\title{
Perfectionism and Impostorism in Becoming a Psychiatrist
}

\author{
Adam M. Brenner ${ }^{1}$ \\ Published online: 30 November 2021 \\ (c) Academic Psychiatry 2021
}

Golden words make practice, practice makes perfect, perfect is a fault, and fault lines change. [1]

Suicide is the second leading cause of death among young people of medical school age [2]. This is a time of life when developmental tasks include consolidating a social and professional identity. Medical students are in the process of succeeding in this challenge, and one might hope that would actually be protective against suicide risk. Instead, we find the opposite to be true. Medical students are at increased risk of suicidality [3]. What can bring such talented, accomplished, and mission-oriented young people to suicide?

Brennan-Wydra and colleagues [4] provide one possible element of the puzzle in their valuable paper, "Maladaptive Perfectionism, Impostor Phenomenon, and Suicidal Ideation Among Medical Students." They surveyed medical students at a single institution and received responses from 226, a rate of return of $41 \%$. Suicidal ideation was assessed by the Suicide Behaviors Questionnaire-Revised (SBQ-R), which examines suicidal ideation over the past year and over a lifetime, suicide threats, and self-report of risk of suicide in the future.

The respondents also completed the Short Almost Perfect Scale (SAPS) [5]. It has eight questions graded on a Likert scale; half of them focus on standards and half on discrepancy between self-perception and those standards. High standards are assessed by statements like "I have a strong need to strive for excellence" and "I set very high standards for myself." Discrepancy is assessed by statements like "Doing my best never seems to be enough" and "I am hardly ever satisfied with my performance." The authors make the distinction between adaptive and maladaptive perfectionism. The former are those with high scores on standards but not on discrepancy; the latter, by high scores on both.

Impostor phenomenon was measured by the brief ( 7 questions) Leary Impostor Scale [6], also a Likert-type

Adam M. Brenner

adam.brenner@utsouthwestern.edu

1 University of Texas Southwestern Medical Center, Dallas, TX, USA assessment. Statements include, for example, "Sometimes I'm afraid others will discover how much knowledge or ability I really lack", "Sometimes I am afraid I will be discovered for who I really am", and "In some situations I feel like a 'great pretender'; that is, I'm not as genuine as others think I am." The statements capture different aspects of feeling like an impostor. There is the sense that I have fooled others into thinking I' $m$ more capable than is true. But there is also the anxiety that I'm not as genuine as I appear. Many medical students are drawn to be physicians because of the idea of being empathically present to their patients. The concern that this is not authentic can be very painful.

The study authors hypothesized that maladaptive perfectionism and suicidality would be correlated, and that impostorism would mediate the relationship. In their model, the pain of falling short of excessively high standards would lead to feeling unworthy of the medical student role and therefore an impostor in filling it. This would in turn create risk for suicidality. The data supported their hypothesis. While these results should be read as preliminary-drawn from a relatively small sample at one medical school-it is intriguing, worth replicating, and important to consider the implications.

The authors suggest that we might use tools such as the SAPS to identify medical students at risk and then enhance their resilience with targeted cognitive behavioral therapy interventions. However, the authors also wisely note that medicine's "culture of perfection" needs to change. We need to help students appreciate that mistakes and uncertainty are inevitabilities for a life in medicine. Unlike a standardized multiple-choice question exam, perfection will not be possible. They call for greater openness in embracing uncertainty, acknowledging errors, and fostering a mindset of lifelong learning and growth. Nowhere is this truer than in psychiatry.

Perhaps in some specialties one could talk of a procedure that went perfectly or a diagnostic interview where nothing was missed. But in psychiatry, our interviews are often our procedures as well-they are both diagnostic and therapeutic - and though we can continue to improve throughout our career, we will not achieve perfection. When I demonstrate 
interviewing therapeutically for medical students, I ask them to point out the moments that were most successful and the moments that were least. Where did I miss some nuance of feeling or hint of meaning? Where did I push too hard, or where was I too passive? These are inevitable. Psychiatric illnesses are too complex-people are too complex-to ever leave an encounter and know that our performance was perfect.

Because psychiatry depends so much on using the physician's own person as an instrument of healing, it can be easy for trainees to feel they are impostors in offering their empathic selves. After all, they know that they still have so much to learn and experience in life. And yet, how often as educators do we see a patient respond to a trainee in a way that they have not responded to anyone else? When this happens, it is because the student or resident allowed some real part of themselves to be present with the patient. Of course, different patients need different things from their psychiatrists, and the range of what one can authentically offer will not always suffice. The point is that there can be no ideal of a generic psychiatrist to compare oneself to. I can only be the psychiatrist that I can be, and I cannot then be an impostor. Who else could be that psychiatrist instead?

Jason Cheng [7] writes from the same perspective, but in terms of psychiatric leadership rather than patient care, in his beautiful Faculty Viewpoint, “The Quiet Leader.” I will close with letting his words speak for themselves:

With our leadership team including our department chair and chief residents, different members connect better with different people and are naturals in different scenarios, which can be mediated by personality or culture. Therefore, our differences in these areas allow us to better handle anything that comes up. If we encourage people of different personalities and cultures to contribute as leaders in medicine, we will be stronger for it.

This is just another way to appreciate how crucial diversity and inclusion are in psychiatry. We need every kind of person if we hope to have each patient find the healer they need, and each team find the leader they need. And then we must make sure that each person we recruit into psychiatry understands that we wanted them not for how they measured up to some ideal of perfection, but for the potential we saw in them to become the imperfect—but unique and crucialpsychiatrist that only they are qualified to become.

\section{Declarations}

Disclosures The author states that there is no conflict of interest.

\section{References}

1. R.E.M. I believe. Lifes Rich Pageant. Belmont, Indiana. 1986.

2. National Institute of Mental Health. Suicide. https://www.nimh. nih.gov/health/statistics/suicide. Accessed 22 Nov 2021.

3. Schwenk TL, Davis L, Wimsatt LA. Depression, stigma, and suicidal ideation in medical students. JAMA. 2010;304(11):1181-90.

4. Brennan-Wydra E, Chung HW, Angoff N, ChenFeng J, Phillips A, Schreiber J, Young C, Wilkins K. Maladaptive perfectionism, impostor phenomenon, and suicidal ideation among medical students. Acad Psychiatry. 2021. https://doi.org/10.1007/ s40596-021-01503-1.

5. Rice KG, Richardson CM, Tueller S. The short form of the revised Almost Perfect Scale. J Pers Assess. 2014;96(3):368-79.

6. Leary MR, Patton KM, Orlando AE, Funk WW. The impostor phenomenon: self-perceptions, reflected appraisals, and interpersonal strategies. J Pers. 2000;68(4):725-56.

7. Cheng JE. The quiet leader. Acad Psychiatry. 2021. https://doi. org/10.1007/s40596-021-01536-6.

Publisher's Note Springer Nature remains neutral with regard to jurisdictional claims in published maps and institutional affiliations. 